\title{
Construction and Validation of a Metabolic Reprogramming Related Prognostic Model for Hepatocellular Carcinoma
}

\section{Xiaohong - Liu}

Wuhan University Second Clinical College: Wuhan University Zhongnan Hospital

https://orcid.org/0000-0001-7731-6634

Qian - Xu

Wuhan University Zhongnan Hospital

Zi-Jing - Li

Wuhan University Zhongnan Hospital

Bin - Xiong ( $\square$ binxiong1961@whu.edu.cn )

Department of Gastrointestinal Surgery \& Department of Gastric and Colorectal Surgical Oncology, Zhongnan Hospital of Wuhan University, Wuhan, Hubei, China.

\section{Research article}

Keywords: metabolic reprogramming, hepatocellular carcinoma, prognosis, The Cancer Genome Atlas, International Cancer Genome Consortium, bioinformatics

Posted Date: October 29th, 2020

DOI: https://doi.org/10.21203/rs.3.rs-97188/v1

License: (c) (i) This work is licensed under a Creative Commons Attribution 4.0 International License. Read Full License 


\section{Abstract}

\section{Background}

Metabolic reprogramming is an important hallmark in the development of malignancies. Numerous metabolic genes have been demonstrated to participate in the progression of hepatocellular carcinoma (HCC). However, the prognostic significance of the metabolic genes in HCC remains elusive.

\section{Methods}

We downloaded the gene expression profiles and clinical information from the GEO, TCGA and ICGC databases. The differently expressed metabolic genes were identified by using Limma R package. Univariate Cox regression analysis and LASSO (Least absolute shrinkage and selection operator) Cox regression analysis were utilized to uncover the prognostic significance of metabolic genes. A metabolism-related prognostic model was constructed in TCGA cohort and validated in ICGC cohort. Furthermore, we constructed a nomogram to improve the accuracy of the prognostic model by using the multivariate Cox regression analysis.

\section{Results}

The high-risk score predicted poor prognosis for HCC patients in the TCGA cohort, as confirmed in the ICGC cohort $(P<0.001)$. And in the multivariate Cox regression analysis, we observed that risk score could act as an independent prognostic factor for the TCGA cohort (HR (hazard ratio) $3.635,95 \% \mathrm{Cl}$ (confidence interval)2.382-5.549) and the ICGC cohort (HR1.905, 95\% Cl 1.328-2.731). In addition, we constructed a nomogram for clinical use, which suggested a better prognostic model than risk score.

\section{Conclusions}

Our study identified several metabolic genes with important prognostic value for HCC. These metabolic genes can influence the progression of $\mathrm{HCC}$ by regulating tumor biology and can also provide metabolic targets for the precise treatment of HCC.

\section{Background}

Hepatocellular carcinoma (HCC) ranks sixth among the most common neoplasms with one of the highest mortality rates among cancers $[1,2]$. And HCC is a highly recurrent and drug-resistant malignancy that is usually diagnosed at an advanced stage [3]. An in-depth recognition of the regulatory mechanisms in tumor development can improve the efficiency of early diagnosis and precise treatment, thus improving the clinical prognosis of HCC patients.

Metabolic reprogramming is recognized as an important hallmark in cancer [4]. It has been reported that metabolic reprogramming provides a selective advantage for the growth and proliferation of tumor cells by modifying their metabolic programs to adapt to their increasing requirements of energy and 
macronutrients [5]. It has the ability to regulate both genetic and epigenetic events in tumor cells [6]. Moreover, cellular metabolic pathways have been reported to influence tumor immunotherapy by regulating $T$ cell function and longevity [7]. And changes in tumor metabolic activity may help identify new forms of communication in the tumor microenvironment [8]. The liver provides a critical platform for many physiological functions including macronutrient metabolism, homeostasis of lipid and cholesterol, immune system support, decomposition of exogenous compounds and so on [9]. The metabolic processes in human hepatocellular carcinoma are also complicated. The upregulated GLUT1 and GLUT2 isoforms in HCC can enhance glucose uptake $[10,11]$. Aberrant changes in lipid metabolism such as phospholipids and fatty acids metabolites were found in serum samples of HCC patients $[12,13]$. And tumor metabolic heterogeneity in $\mathrm{HCC}$ can also affect the bone marrow-derived inhibitory cells (MDSC) and tumor-related macrophages (TAM) in the tumor microenvironment [14-16]. Therefore, metabolic reprogramming is of great significance in $\mathrm{HCC}$, and its regulatory directions are extensive and complex. However, the underlying mechanisms of metabolic abnormalities in the development of HCC remain unclear. Nevertheless, few studies have systematically investigated the metabolic reprogramming and its relationship with prognosis in HCC.

In this study, we analyzed the high-throughput data of HCC to systematically investigate the metabolic changes and their potential regulatory mechanisms in hepatocellular carcinoma. And we also performed LASSO Cox regression analysis to select a gene set with the most prognostic value to construct a metabolism-related prognostic model, thus shedding a light into the clinical significance and application value of the metabolic reprogramming.

\section{Materials And Methods}

\subsection{Microarray Datasets}

The gene expression matrix files (including $183 \mathrm{HCC}$ samples and 15 adjacent noncancerous samples) from GSE112790 based on platform GPL570 were downloaded from the Gene Expression Omnibus (GEO) database (https://www.ncbi.nlm.nih.gov/geo/).

\subsection{RNA-Sequencing Datasets}

The gene expression data and the corresponding clinical information of HCC were downloaded from The Cancer Genome Atlas (TCGA) website (https://portal.gdc.cancer.gov/) and the International Cancer Genome Consortium (ICGC) website (https://icgc.org/). 424 TCGA samples from the United States and 445 ICGC samples from Japan were further studied. The log2 transformations were performed for all the expression data. The gene expression data and the corresponding clinical information are publicly available and analyzed under the guiding of pertinent instructions and regulations.

2.3 Differentially Expressed Genes (DEGs) Analysis 
We used the limma R package to determine the DEGs of HCC cohorts in TCGA, ICGC and GSE112790. By studying metabolism-related signaling pathways in GSEA software(c2.cp.kegg.v7.0.symbols.gmt), we screened out 944 metabolic genes. The metabolism-related DEGs were obtained by intersecting the previously obtained DEGs with metabolic genes.

\subsection{Functional and Pathway Enrichment Analysis}

Gene annotation enrichment analysis was performed by the clusterProfiler R package to investigate the metabolism-related DEGs at the functional level. Metabolism-related biological processes were identified by Gene Ontology (GO) analysis. And Kyoto Encyclopedia of Genes and Genomes (KEGG) analysis identified the metabolism-related pathways. The analyses were performed with the cutoff of $p<0.05$ and $\mathrm{q}<0.05$, false discovery rate $(F D R)<0.05$.

\subsection{Construction and Validation of a Metabolic Prognostic Model}

We calculated the HR of DEGs in the TCGA and ICGC cohorts by using the univariate Cox regression analysis $(P<0.05)$. Then we used LASSO Cox regression analysis to screen out the genes with the most prognostic value in DEGs. The formula that risk score $=\Sigma$ cox gene coefficient of gene Xi $\times$ scale express the value of $X i$ was applied to establish a risk score model to predict the patient survival. The HCC cohorts with integrate clinical information were analyzed to determine independent prognostic factors through multivariate Cox regression analysis. Finally, the area under the curve (AUC) of receiver Operating characteristic (ROC) curve was quantified by the timeROC R package to show the sensitivity and specificity of risk score in predicting the overall survival for HCC. We also established a Nomogram to show the prognostic value of risk scores by using the coefficients of multivariate Cox regression analysis. Concordance index (C-index) was calculated and used to evaluate the predictive accuracy of the prognostic model and the nomogram. Moreover, we plotted the calibration curves to appraise the predicting ability of the nomogram through the rms $\mathrm{R}$ package.

2.6 Correlation analysis of metabolism-related genes and tumor immunity in the TCGA HCC cohort.

We obtained the immune related genes from immune-related signaling pathways in GSEA software and performed the correlation analysis between risk genes and immune related genes. Then we utilized GSVA and GSEABase R packages to perform ssGSEA analysis on the TCGA cohort. Through limma and sparcl R packages, we clustered the HCC cohort into high, medium and low immune score groups, and used limma R package to identify the DEGs between high and low immune score groups. The immune DEGs and metabolism-related genes were intersected to obtain metabolic genes in the high and low immune score groups. Then, the metabolism genes related to immune grouping were subjected to GO and KEGG analyses in the Metascape website (https://metascape.org/gp/index.html), and their correlations with immune infiltration were obtained in the TIMER website (https://cistrome.shinyapps.io/timer/).

\subsection{Statistical Analysis}


R software was used to conduct all the statistical analyses. The statistical significance of two normally distributed variables was accessed by unpaired t-test and the correlation between the gene sets was determined with Spearman's test. Kaplan-Meier method was used to compare the survival curves of each subgroup, and log-rank test was used to determine the statistical significance of individual variable in survival. All statistical tests were two-tailed and $p<0.05$ was considered statistically significant.

\section{Results}

3.1 Identification of metabolism-related DEGs between HCC samples and normal samples.

In order to identify metabolism-related DEGs between $\mathrm{HCC}$ and normal tissues, we conducted differential expression analysis in TCGA(LICH), ICGC(LIRI-JP) and GSE112790 datasets by using the limma $R$ package ( $F D R<0.05$ and $\mid \log 2 \mathrm{FCl}>1)$. We obtained $457 \mathrm{DEGs}$, of which 100 were related to metabolic reprogramming. (Supplementary Fig.S1 and Supplementary Table S1).

\subsection{GO and KEGG analyses in metabolism-related genes.}

To gain further insight into the function of the identified metabolism-related DEGs, we performed $G O$ and KEGG analyses. The DEGs were mainly involved in biological processes associated response to xenobiotic stimulus, nucleoside phosphate biosynthetic process, carboxylic acid biosynthetic process and so on (Fig. 1a). Moreover, the DEGs were involved in multiple KEGG pathways, including retinol metabolism, drug metabolism-cytochrome P450, arachidonic acid metabolism, et al (Fig. 1b).

3.3 Construction and validation of a metabolism-related prognostic model in the TCGA and ICGC cohorts.

Considering the difference in the expression of metabolism related genes between HCC and normal tissues, we determined to analyze the clinical application value of the DEGs in depth. Among the 100 metabolic DEGs, 37 genes were demonstrated to be significantly associated with the overall survival status of HCC patients through the univariable Cox regression analysis (Supplementary Fig. S2a), and 9 of them were identified to have the maximum prognostic value through the LASSO Cox regression analysis (Supplementary Figs. S2b,c ). Finally, we applied these genes to establish a metabolism-related model in the TCGA cohort to estimate the prognostic value of metabolic reprogramming in HCC patients. And the identical formula was utilized in the ICGC cohort to determine whether the prognostic model was reliable in different populations (Figs. 2a-f). The formula for the prognostic model was that risk score $=\Sigma$ Cox coefficient of gene Xi $\times$ scale expression value of gene Xi. Then, HCC patients were categorized into high- or low- risk groups with the optimal cut-off value of risk score. The high-risk score predicted poor prognosis for HCC patients in the TCGA cohort $(P=1.847 \mathrm{e}-07)$, as confirmed in the ICGC cohort $(P=$ 7.243e-06). And we found that the risk score in TCGA cohort had excellent prognostic value in both univariate and multivariate Cox regression analyses, which was consistent with the results in ICGC cohort (Figs. 3a-d). The results showed that metabolic alterations had important clinical significance in the prognosis of HCC patients. And the risk score of the metabolism-related prognostic model can act as an 
independent prognostic factor for the TCGA cohort (HR (hazard ratio) 3.635, 95\% Cl (confidence interval)2.382-5.549) and the ICGC cohort (HR1.905, 95\% Cl 1.328-2.731) in HCC patients.

3.4 Evaluation of the metabolism-related prognostic model in the TCGA and ICGC HCC cohorts.

To further evaluate the prognostic accuracy of the metabolism-related prognostic model, we calculated the area under ROC curve (AUC) and the C-index value of the model (Figs. 4a-f). And we also constructed a nomogram with comprehensive consideration of risk score and clinical characteristics for clinical use (Fig. 4g). We found that the AUC of the prognostic model on OS status was 0.758 at 1 year, 0.742 at 2 years, 0.693 at 3 years, 0.7 at 4 years and 0.693 at 5 years in TCGA cohort, while 0.749 at 1 year, 0.748 at 2 years, 0.763 at 3 years, 0.83 at 4 years and 0.672 at 5 years in ICGC cohort (Figs. $4 a, d)$. We also found the overall AUC (0.755) of risk score was superior to that of grade (0.478), stage (0.698), T (0.704), N (0.506), M (0.508) in TCGA cohort. And the overall AUC (0.749) of risk score in ICGC cohort was superior to that of priorMalignancy (0.526), indicating that the prognostic model was well constructed (Figs. 4b,e). In addition, through principal component analysis (PCA) of unsupervised learning, we found that HCC samples were well distinguished between high and low risk grouping in the predictive model (Figs. 4c,f). Furthermore, we observed that the C-index of risk score was 0.7260 in TCGA cohort and 0.7195 in ICGC cohort. And the C-index of nomogram was 0.7510 in TCGA cohort and 0.8240 in ICGC cohort, indicating higher predictive accuracy of nomogram than risk scores. Finally, we conducted the calibration curves at 3 years and at 5 years, to validate the nomogram's performance and observed that the predictive curves worked well (Figs. 4h,i).

3.5 Clinical relevance of metabolism-related prognostic model and its constructed genes in TCGA cohort

We conducted a clinical correlation analysis for risk score of the prognostic model to further understand the influence of metabolic reprogramming on the clinical prognosis of HCC patients. The results showed that risk score had significant correlations with the status $(p=1.11 \mathrm{e}-06)$, grade $(p=0.003)$, stage $(p=0.014)$ and T $(p=0.013)$ in HCCs in TCGA cohort (Figs. $5 a-d)$. The results suggested that Risk score correlates with the progression of hepatocellular carcinoma. And a higher risk score indicates a higher degree of malignancy in HCC. Therefore, metabolic alterations are closely related to the clinical prognosis of HCC patients.

3.6 The role of risk score in the prognosis of HCC patients stratified by clinicopathological variables

To explore the prognostic value of risk score in HCC patients stratified by different clinicopathological variables, we stratified HCC patients by age, sex, grade, stage and TNM stage. The results showed that the high-risk group had a poor prognosis among patients stratified by different clinical variables (Fig. 6). These results suggested that metabolism-related prognostic risk score was not limited by clinicopathological variables in predicting the prognosis of HCC patients.

3.7 Correlation between metabolism-related genes and tumor immunity in the TCGA HCC cohort. 
Since our analysis found that there was a significant correlation between the expression of metabolismrelated risk genes and immune-related genes, we further analyzed the metabolic alterations in the high and low immune-rating groups (Supplementary Figs. S4a,b). And we found that risk score was significantly higher in the low immune group than in the high immune group (Supplementary Fig. S4e). There were 23 metabolism-related genes overexpressed in the high immune score group, while 37 in the low immune score group (FDR $<0.05$ and $\mid \log 2 \mathrm{FCl}>1$, Supplementary Figs.S4c,d). By GO and KEGG analyses, it was found that the metabolic genes in the high immune score group were mainly involved in the organophosphate biosensitization and arachidonic acid signaling pathway, et al. While in the low immune score group, the metabolic genes were mainly enriched in lipid biosensitization, glutathione metabolism and so on (Figs. 7a,b). We also found that there was a close relationship between metabolism-related genes and tumor immune infiltration, especially in the high immune score group (Supplementary Fig.S5). We speculated that metabolism-related genes may indirectly regulate tumor immunity through metabolic alterations to some extent, thus affecting the prognosis of HCC patients.

\section{Discussion}

Metabolic reprogramming plays an important role in the development of HCC [17]. Patients with reduced serum albumin levels had larger and more aggressive tumors in HCC [18]. HCC cells sustain the antioxidant reaction by delivering glucose into the pentose phosphate pathway through ROS-mediated PKM2 inhibition [19]. And TAM used the lactic acid released by the HCC cells to induce tumor stem celllike characteristics and drug resistance [20]. Therefore, further studies on the role of metabolic reprogramming in $\mathrm{HCC}$ development and its prognostic value are urgent. And our study provides insights into the underlying mechanisms of metabolic alterations and their clinical prognostic significance in hepatocellular carcinoma.

In this study, we screened out 100 metabolism-related DEGs to explore the metabolic alterations in the development of HCC. And the results of gene annotation enrichment analyses suggested that metabolism-related DEGs were enriched in the biological process, including response to xenobiotic stimulus, xenobiotic metabolic process, nucleoside phosphate biosynthetic process and carboxylic acid biosynthetic process, et al (Fig. 1a). Previous studies have shown that these biological processes are indeed involved in the development and progression of hepatocellular carcinoma by mediating druginduced liver disease, affecting DNA replication and repair, RNA synthesis, and acting as potential pharmacophore [21-23]. And the KEGG analysis revealed that metabolism-related DEGs were involved in pathways like retinol metabolism, drug metabolism, metabolism of xenobiotics by cytochrome P450, glycolysis/gluconeogenesis, fatty acid degradation and tyrosine metabolism, et al (Fig. 1b). This suggested that metabolic reprogramming may mediate tumorigenesis, metastasis, and tumor resistance in HCC by these pathways, which was consistent with the current researches [24-26]. In particular, glycolytic related genes such as ALDH3A1, ALDOA were up-regulated in HCC compared with normal tissues, while the expression of gluconeogenic related genes such as FBP1 and PCK1 were decreased. Enhanced glycolysis in hepatocellular carcinoma can be explained as metabolic adaptation to the tumor microenvironment. In addition, our findings were consistent with those of Curtis C, et al [27], in which 
glycosoplasmas were transferred to regulate oxidative stress and countered SAM elevation, thereby regulating hepatocellular carcinoma biosynthetic pathways.

In the retrospective analysis, we constructed and verified a metabolism-related model to predict the prognosis for HCC patients (Fig. 2). The results showed that there was a significant difference in overall survival between the high- and low-risk groups. Moreover, through univariable and multivariable Cox regression analyses, we found that risk score could be used as an independent prognostic factor for HCC, and its prognostic value was similar or even superior to some traditional clinical characteristics to a certain extent. The 9 metabolism-related genes that constituted our prognostic model were RRM2, G6PD, HMOX1, LCAT, AKR1B10, CYP2C9, HMGCS2, TK1 and DPYS, which covered key enzymes of various metabolic pathways. It has been demonstrated that ribonucleoside diphosphate is responsible for transforming ribonucleoside diphosphate into 2'-deoxyribonucleoside, and the ribonucleoside reductase subunit M2 (RRM2) is a therapeutic target for HCC [28]. Up-regulated G6PD can promote the migration and invasion of HCC cells by inducing epithelial-mesenchymal transformation [29]. And HMOX1 influences hepatocellular carcinoma progression by regulating iron metabolism [30]. LCAT is hypermethylated in HCC and can affect the progression of HCC [31]. Immunohistochemical staining with AKR1B10 showed a negative correlation with tumor proliferation in HCC [32]. As one of the most critical drug metabolic enzymes, CYP2C9 regulates the metabolic processes of many carcinogens and drugs in HCC [33]. HMGCS2 catalyzes the formation of HMG-CoA in HCC [34]. And TK1 can catalyze the generation of deoxythymine monophosphate (dTMP) and promote the proliferation of HCC and other malignancies [35]. Among the 9 risk genes, the relationship between DPYS and the development of HCC has not been reported. DPYS can affect the levels of dihydrouracil and dihydrothymine by regulating the metabolism of dihydropyrimidine, which is related to cell transformation and cancer progression [36]. Furthermore, we plotted the receiver operator characteristic curves (ROC) and calculated the AUC value to evaluate the sensitivity and specificity of the prognostic model (Fig. 4). The results suggested that the AUC value of risk score in the TCGA cohort was greater than that of age, gender, grade, stage and TNMstaging. This further demonstrated the clinical application value of this metabolism-related prognostic model. Finally, by establishing a nomogram and comparing the C-Index values, we found that comprehensive consideration of risk score and other traditional clinical features could significantly improve the prognostic accuracy. However, there are still some limitations in our study. For example, the patient's gene expression data and the corresponding clinical characteristics are all publicly available, which are often not comprehensive enough, and more prospective studies need to be carried out clinically. In addition, although we used hepatocellular carcinoma data from the United States and Japan for comparative analysis, more regional datasets need to be included to eliminate geographic differences.

By exploring the differential expression of metabolic genes between the high and low immune score groups as well as the biological processes and signaling pathways involved, we observed that metabolic genes in the high immune score group participated in the NAD metabolic process, regulation of leukocyte apoptosis process, arachidonic acid metabolism and so on, while metabolic genes in the low immune score group were mainly involved in the glutathione metabolism, alcohol metabolism process, et al (Fig. 7). These results were consistent with the existing studies such as nicotinamide adenine 
dinucleotide (NAD+) regulated the immune function of macrophage in inflammation [37], and Arachidonic acid (AA) and its derivatives mediated inflammatory responses [38]. Activated T cells can

resist the oxidation of glutathione (GSH) and prevent oxidative damage [39]. It can protect host immune cells through antioxidant mechanisms and plays an active role in immunocompromised states [40]. Alcohol-mediated oxidative stress can disrupt immune signaling pathways and increase the risk of immune system dysfunction [41]. However, oxidative metabolites of ethanol can induce Kupffer cells to release proinflammatory cytokines, promoting neutrophil infiltration [42]. This indicated that the mechanism and direction of alcohol metabolism regulating immunity were complex in the immunocompromised state. Moreover, metabolic genes in the high immune score group were significantly correlated with immune infiltrating cells in $\mathrm{HCC}$, which further demonstrated the interaction between metabolic changes and the tumor immunity. These results suggested that metabolic reprogramming may affect the tumor microenvironment in HCC to some degree.

\section{Conclusions}

In summary, we systematically analyzed metabolic alterations in HCC through bioinformatic methods in this study. This increases our understanding of the impact of metabolic reprogramming on tumorigenesis and progression. Then, we established and verified a metabolism-related prognostic model by using LASSO analysis to clarify the clinical significance of metabolism-related genes in the prognosis of HCC. It shed a new light into the metabolic prospective in predicting the prognosis of $\mathrm{HCC}$, which is helpful to further investigate the potential metabolic targets in the clinical treatment of HCC.

\section{Abbreviations}

HCC

hepatocellular carcinoma

TCGA

The Cancer Genome Atlas

ICGC

International Cancer Genome Consortium

GEO

Gene Expression Omnibus

DEG

Differentially Expressed Genes

LASSO

Least absolute shrinkage and selection operator

PCA

principal component analysis

AUC

area under ROC curve 


\section{Declarations}

\section{Availability of data and materials}

The datasets analyzed for this study can be found in The Cancer Genome Atlas

(https://portal.gdc.cancer.gov/), International Cancer Genome Consortium (https://icgc.org/) and the Gene Expression Omnibus (https://www.ncbi.nlm.nih.gov/geo/).

\section{Ethics approval and consent to participate}

Not applicable.

\section{Consent for publication}

Not applicable.

\section{Competing interests}

The authors declare that the research was conducted in the absence of any commercial or financial relationships that could be construed as a potential conflict of interest.

\section{Funding}

This work was supported by the National Natural Science Foundation of China under Grant [number 81572874, 81702411 and 81872376]; and Health Commission of Hubei Province Scientific Research Project under Grant [number WJ2019H012].

\section{Author contributions}

All of the authors read and approved the final manuscript. Bin Xiong conceived the work. Xiaohong Liu performed the analysis, collated the results and wrote the manuscript. Qian Xu checked the associated database and raw data. Zijing Li revised the manuscript.

\section{Contributor Information}

Xiao-Hong Liu, Email: 2015302180120@whu.edu.cn.

Qian Xu, Email: whuxuq@whu.edu.cn.

Zi-Jing Li, Email: lizijing7@126.com.

Bin Xiong, Email: binxiong1961@whu.edu.cn.

\section{Acknowledgements}

We thank Xingang Peng and Mingyu Sun for technology support. 


\section{References}

1. Forner A, Reig M, Bruix J. Hepatocellular carcinoma. Lancet. 2018;391(10127):1301-1314. doi: 10.1016/S0140-6736(18)30010-2.

2. El-Serag HB. Epidemiology of viral hepatitis and hepatocellular carcinoma. Gastroenterology. 2012;142(6):1264-1273.e1. doi: 10.1053/j.gastro.2011.12.061.

3. Matteis SD, Ragusa A, Marisi G, et al. Aberrant Metabolism in Hepatocellular Carcinoma Provides Diagnostic and Therapeutic Opportunities. Oxid Med Cell Longev. 2018; 2018:7512159. doi: 10.1155/2018/7512159. eCollection 2018.

4. Hanahan D, Weinberg RA. Hallmarks of cancer: the next generation. Cell. 2011;144(5):646-74. doi: 10.1016/j.cell.2011.02.013.

5. Peng X, Chen ZY, Farshidfar F, et al. Molecular Characterization and Clinical Relevance of Metabolic Expression Subtypes in Human Cancers. Cell Rep. 2018;23(1):255-269.e4. doi: 10.1016/j.celrep.2018.03.077.

6. Hirschey MD, DeBerardinis RJ, Diehl AME, et al. Dysregulated metabolism contributes to oncogenesis. Semin Cancer Biol. 2015;35 Suppl:S129-S150. doi: 10.1016/j.semcancer.2015.10.002.

7. Kishton RJ, Sukumar M, Restifo NP. Metabolic regulation of T cell longevity and function in tumor immunotherapy. Cell Metab. 2017;26(1):94-109. doi: 10.1016/j.cmet.2017.06.016.

8. Reina-Campos M, Moscat J, Diaz-Meco M. Metabolism Shapes the Tumor Microenvironment. Curr Opin Cell Biol. 2017; 48:47-53. doi: 10.1016/j.ceb.2017.05.006.

9. Trefts E, Gannon M, Wasserman DH. The Liver. Curr Biol. 2017;27(21):R1147-R1151. doi: 10.1016/j.cub.2017.09.019.

10. Parikh U, Marcus C, Sarangi R, Taghipour M, Subramaniam RM. FDG PET/CT in pancreatic and hepatobiliary carcinomas: value to patient management and patient outcomes. PET Clinics PET Clin. 2015;10(3):327-343. doi: 10.1016/j.cpet.2015.03.001.

11. Grobholz R, Hacker HJ, Thorens B, Bannasch P. Reduction in the expression of glucose transporter protein GLUT 2 in preneoplastic and neoplastic hepatic lesions and reexpression of GLUT 1 in late stages of hepatocarcinogenesis. Cancer Res. 1993;53(18):4204-11.

12. Chen $T L$, Xie GX, Wang $X Y$, et al. Serum and urine metabolite profiling reveals potential biomarkers of human hepatocellular carcinoma. Mol Cell Proteomics. 2011;10(7):M110.004945. doi: 10.1074/mcp.M110.004945.

13. Yamada S, Takashina $\mathrm{YK}$, Watanabe $\mathrm{M}$, et al. Bile acid metabolism regulated by the gut microbiota promotes non-alcoholic steatohepatitis-associated hepatocellular carcinoma in mice. Oncotarget. 2018;9(11):9925-9939. doi: 10.18632/oncotarget.24066.

14. Biswas SK, Allavena P, Mantovani A. Tumor-associated macrophages: functional diversity, clinical significance, and open questions. Semin Immunopathol. 2013;35(5):585-600. doi: 10.1007/s00281013-0367-7. 
15. Budhu A, Forgues $\mathrm{M}, \mathrm{Ye} \mathrm{QH}$, et al. Prediction of venous metastases, recurrence, and prognosis in hepatocellular carcinoma based on a unique immune response signature of the liver microenvironment. Cancer Cell. 2006;10(2):99-111. doi: 10.1016/j.ccr.2006.06.016.

16. Husain Z, Huang Y, Seth P, Sukhatme VP. Tumor-derived lactate modifies antitumor immune response: effect on myeloid-derived suppressor cells and NK cells. J Immunol. 2013;191(3):1486-95. doi: 10.4049/jimmunol.1202702.

17. Pavlova NN, Thompson CB. The Emerging Hallmarks of Cancer Metabolism. Cell Metab. 2016;23(1):27-47. doi: 10.1016/j.cmet.2015.12.006.

18. Liou GY, Storz P. Reactive oxygen species in cancer. Free Radic Res. 2010;44(5):479-96. doi: 10.3109/10715761003667554.

19. Biswas SK, Sica A, Lewis CE. Plasticity of macrophage function during tumor progression: regulation by distinct molecular mechanisms. J Immunol. 2008;180(4):2011-7. doi: 10.4049/jimmunol.180.4.2011.

20. Chang $\mathrm{CH}$, Qiu J, O'Sullivan D, et al. Metabolic Competition in the Tumor Microenvironment Is a Driver of Cancer Progression. Cell. 2015;162(6):1229-41. doi: 10.1016/j.cell.2015.08.016.

21. Österreicher $\mathrm{CH}$, Trauner M. Xenobiotic-induced Liver Injury and Fibrosis. Expert Opin Drug Metab Toxicol. 2012;8(5):571-80. doi: 10.1517/17425255.2012.674511.

22. Johnson DC, Widlanski TS. Overview of the Synthesis of Nucleoside Phosphates and Polyphosphates. Curr Protoc Nucleic Acid Chem. 2004;13:13.1. doi:

10.1002/0471142700.nc1301s15.

23. Ballatore C, Huryn DM, Smith AB. Carboxylic Acid (Bio)isosteres in Drug Design. ChemMedChem. 2013;8(3):385-95. doi: 10.1002/cmdc.201200585.

24. Pettinelli P, Arendt BM, Teterina A, et al. Altered Hepatic Genes Related to Retinol Metabolism and Plasma Retinol in Patients With Non-Alcoholic Fatty Liver Disease. PLoS One. 2018;13(10):e0205747. doi: 10.1371/journal.pone.0205747.

25. Hu DG, Marri S, McKinnon RA, Mackenzie PI, Meech R. Deregulation of the Genes That Are Involved in Drug Absorption, Distribution, Metabolism, and Excretion in Hepatocellular Carcinoma. J Pharmacol Exp Ther. 2019;368(3):363-381. doi: 10.1124/jpet.118.255018.

26. Li JB, Huang QC, Long XY, et al. CD147 Reprograms Fatty Acid Metabolism in Hepatocellular Carcinoma Cells Through Akt/mTOR/SREBP1c and P38/PPARa Pathways. J Hepatol. 2015;63(6):1378-1389. doi: 10.1016/j.jhep.2015.07.039.

27. Hughey CC, Trefts E, Bracy DP, et al. Glycine N-methyltransferase deletion in mice diverts carbon flux from gluconeogenesis to pathways that utilize excess methionine cycle intermediates. J Biol Chem. 2018;293(30):11944-11954. doi: 10.1074/jbc.RA118.002568.

28. 28. Yang PM, Lin LS, Liu TP. Sorafenib Inhibits Ribonucleotide Reductase Regulatory Subunit M2 (RRM2) in Hepatocellular Carcinoma Cells. Biomolecules. 2020;10(1):117. doi: 10.3390/biom10010117. 
29. Lu M, Lu L, Dong QZ, et al. Elevated G6PD Expression Contributes to Migration and Invasion of Hepatocellular Carcinoma Cells by Inducing Epithelial-Mesenchymal Transition. Acta Biochim Biophys Sin (Shanghai). 2018;50(4):370-380. doi: 10.1093/abbs/gmy009.

30. Shen Y, Li X, Zhao B, et al. Iron Metabolism Gene Expression and Prognostic Features of Hepatocellular Carcinoma. J Cell Biochem. 2018;119(11):9178-9204. doi: 10.1002/jcb.27184.

31. Long JY, Chen P, Lin JZ, et al. DNA methylation-driven genes for constructing diagnostic, prognostic, and recurrence models for hepatocellular carcinoma. Theranostics. 2019;9(24):7251-7267. doi: 10.7150/thno.31155.

32. Teramoto $R$, Minagawa $H$, Honda $M$, et al. Protein expression profile characteristic to hepatocellular carcinoma revealed by 2D-DIGE with supervised learning. Biochim. Biophys. Acta. 2008;1784(5):764772. doi: 10.1016/j.bbapap.2008.02.011.

33. Yu DK, Green B, Marrone A, et al. Suppression of CYP2C9 by microRNA hsa-miR-128-3p in Human Liver Cells and Association with Hepatocellular Carcinoma. Sci Rep. 2015; 5:8534. doi: $10.1038 /$ srep08534.

34. Wang YH, Liu CL, Chiu WC, Twu YC, Liao YJ. HMGCS2 Mediates Ketone Production and Regulates the Proliferation and Metastasis of Hepatocellular Carcinoma. Cancers (Basel). 2019;11(12):1876. doi: $10.3390 /$ cancers 11121876 .

35. Yeh HW, Lee SS, Chang CY, Hu CM, Jou YS. Pyrimidine metabolic rate limiting enzymes in poorlydifferentiated hepatocellular carcinoma are signature genes of cancer stemness and associated with poor prognosis. Oncotarget. 2017;8(44):77734-77751. doi: 10.18632/oncotarget.20774.

36. Basbous J, Aze A, Chaloin L, et al. Dihydropyrimidinase Protects From DNA Replication Stress Caused by Cytotoxic Metabolites. Nucleic Acids Res. 2020;48(4):1886-1904. doi: 10.1093/nar/gkz1162.

37. Minhas PS, Liu L, Moon PK, et al. Macrophage de novo NAD (+) synthesis specifies immune function in aging and inflammation. Nat Immunol. 2019;20(1):50-63. doi: 10.1038/s41590-018-0255-3.

38. Lee JM, Lee H, Kang S, Park WJ. Fatty Acid Desaturases, Polyunsaturated Fatty Acid Regulation, and Biotechnological Advances. Nutrients. 2016;8(1):23. doi: 10.3390/nu8010023.

39. Mak TW, Grusdat M, Duncan GS, et al. Glutathione Primes T Cell Metabolism for Inflammation. Immunity. 2017;46(4):675-689. doi: 10.1016/j.immuni.2017.03.019.

40. Camila R, Percival SS. Immunomodulatory Effects of Glutathione, Garlic Derivatives, and Hydrogen Sulfide. Nutrients. 2019;11(2):295. doi: 10.3390/nu11020295.

41. Muralidharan S, Ambade A, Fulham MA, et al. Moderate alcohol induces stress proteins HSF1 and hsp70 and inhibits pro-inflammatory cytokines resulting in endotoxin tolerance. J Immunol. 2014;193(4):1975-1987. doi: 10.4049/jimmunol.1303468.

42. Elisabetta C, Tommaso M, Andrea G. Pathogenesis of Alcoholic Liver Disease: Role of Oxidative Metabolism. World J Gastroenterol. 2014;20(47):17756-72. doi: 10.3748/wjg.v20.i47.17756.

\section{Figures}


a

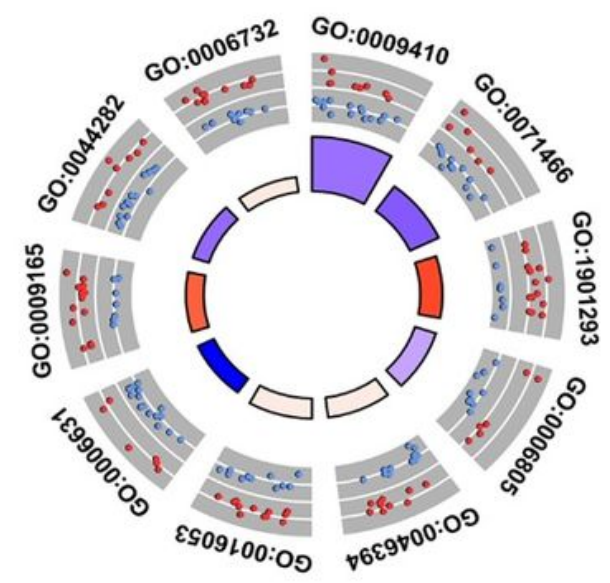

$\underbrace{z \text {-score }}_{\text {decreasing }} \cdot \begin{gathered}\log F \mathrm{C} \\ \text { increasing }\end{gathered}$

b
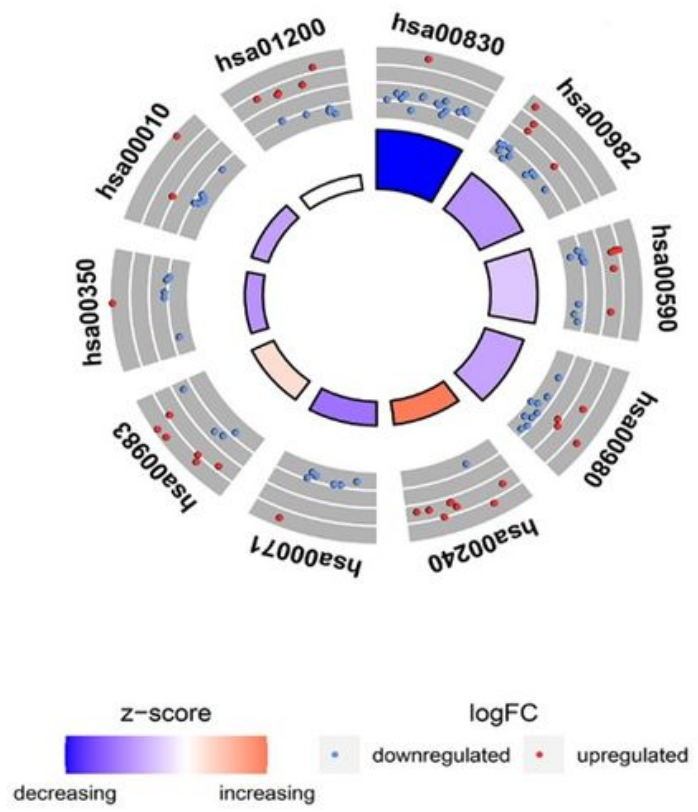

\begin{tabular}{|c|c|}
\hline ID & Description \\
\hline GO:0009410 & response to xenobiotic stimulus \\
\hline GO:0071466 & cellular response to xenobiotic stimulus \\
\hline GO:1901293 & nucleoside phosphate biosynthetic process \\
\hline GO:0006805 & xenobiotic metabolic process \\
\hline GO:0046394 & carboxylic acid biosynthetic process \\
\hline GO:0016053 & organic acid biosynthetic process \\
\hline GO:0006631 & fatty acid metabolic process \\
\hline GO:0009165 & nucleotide biosynthetic process \\
\hline GO:0044282 & small molecule catabolic process \\
\hline GO:0006732 & coenzyme metabolic process \\
\hline
\end{tabular}

\begin{tabular}{|r|r|}
\hline ID & $\begin{array}{c}\text { Description } \\
\text { hsa00830 }\end{array}$ \\
\hline hsa00982 & Rrug metabolism - cytochrome P450 \\
\hline hsa00590 & Arachidonic acid metabolism \\
\hline hsa00980 & Metabolism of xenobiotics by cytochrome P450 \\
\hline hsa00240 & Pyrimidine metabolism \\
\hline hsa00071 & Fatty acid degradation \\
\hline hsa00983 & Drug metabolism - other enzymes \\
\hline hsa00350 & Tyrosine metabolism \\
\hline hsa00010 & Glycolysis / Gluconeogenesis \\
\hline hsa01200 & Carbon metabolism \\
\hline
\end{tabular}

\section{Figure 1}

Functional enrichment analyses of the 100 differentially expressed metabolism-related genes. (a) Circos plot of Gene Ontology (GO) analysis. (b) Circos plot of Kyoto encyclopedia of genes and genomes (KEGG) analysis. The inner circle represents the z-score values, and the outer circle represents the number of genes enriched in the pathway. Red indicates upregulated metabolism-related genes, and blue indicates downregulated metabolism-related genes. 
a

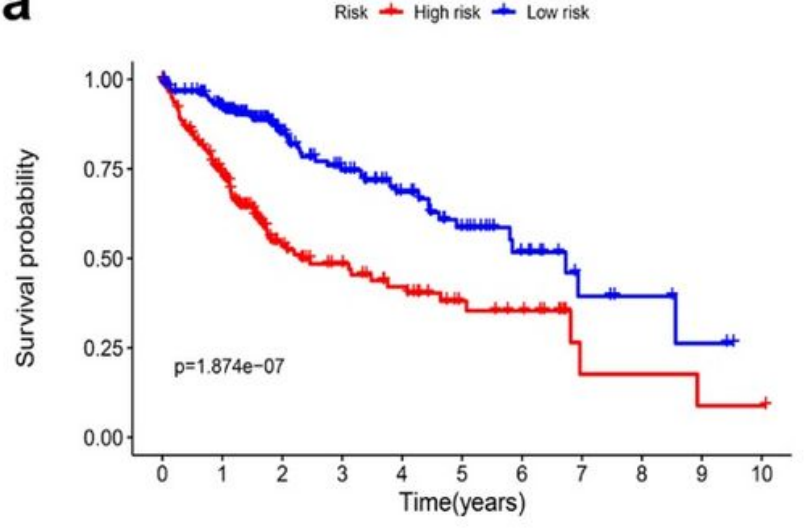

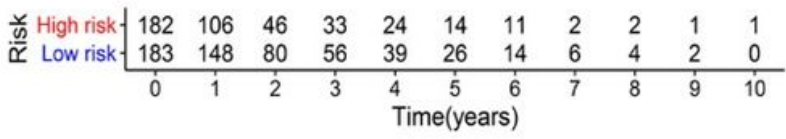

d

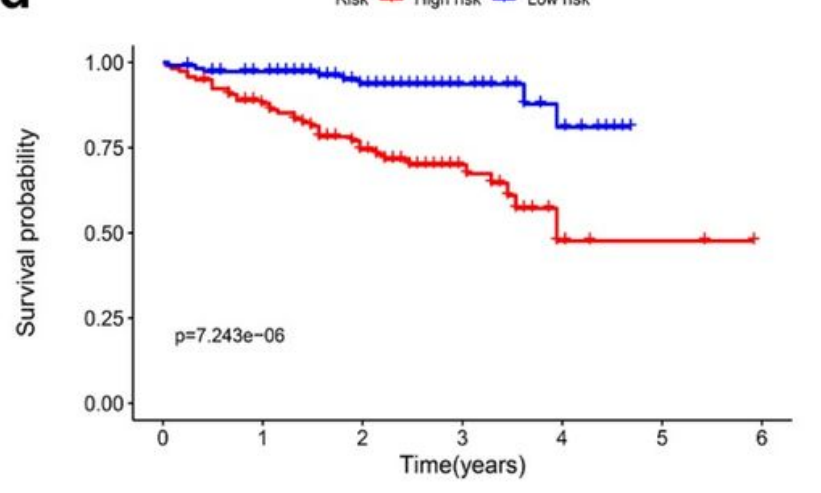

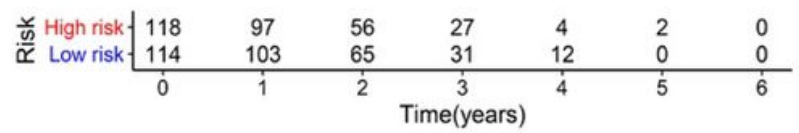

b
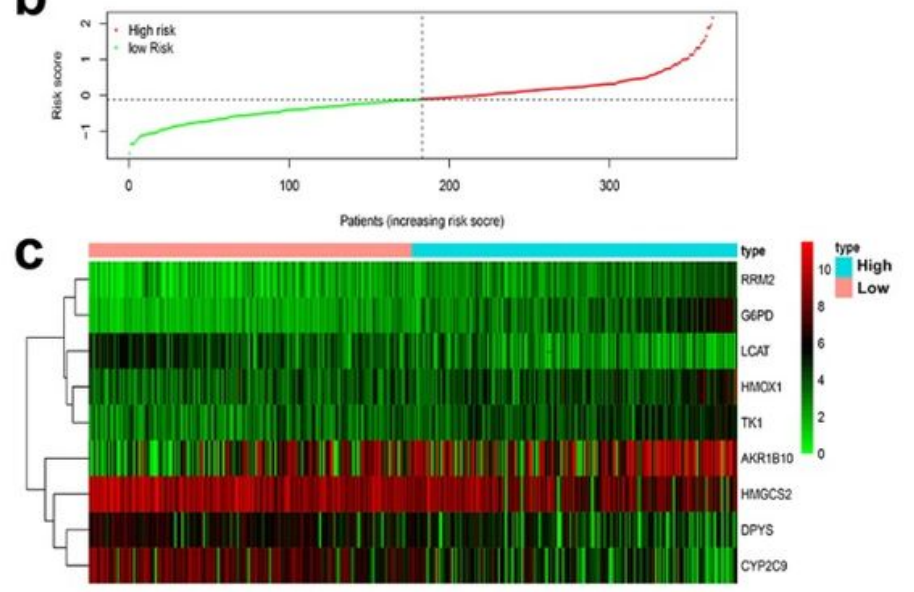

e
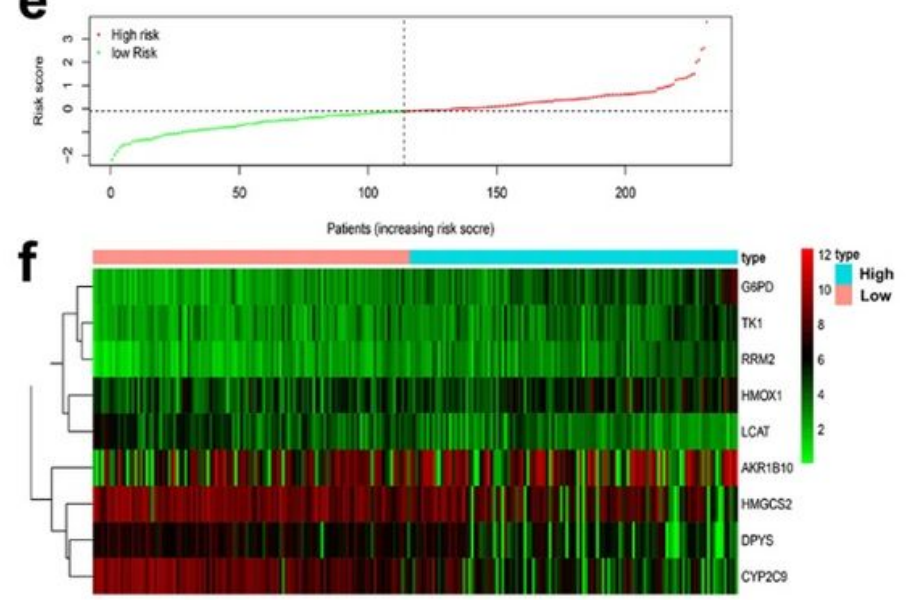

\section{Figure 2}

Construction and validation of a metabolism-related prognostic model in TCGA and ICGC cohorts. (a, d) Kaplan-Meier OS curves for the high- and low-risk groups. OS was significantly higher in the low-risk group than in the high-risk group. (b, e) Distribution of the risk scores of HCC patients. (c, f) Relationship between the risk score and the heatmap for the expression of the selected prognostic genes. OS, overall survival. 
a

$\begin{array}{lrr} & \text { pvalue } & \begin{array}{r}\text { Hazard ratio } \\ \text { age }\end{array} \\ \text { gender } & 0.591 & 1.005(0.987-1.023) \\ \text { grade } & 0.301 & 0.780(0.487-1.249) \\ \text { stage } & <0.914 & 1.017(0.746-1.387) \\ \text { T } & <0.001 & 1.865(1.456-2.388) \\ \text { M } & 0.023 & 3.8050(1.207-12.281) \\ \text { N } & 0.328 & 2.022(0.494-8.276) \\ \text { AFP } & <0.001 & 1.000(1.000-1.001) \\ \text { riskScore } & <0.001 & 3.777(2.615-5.457)\end{array}$

C
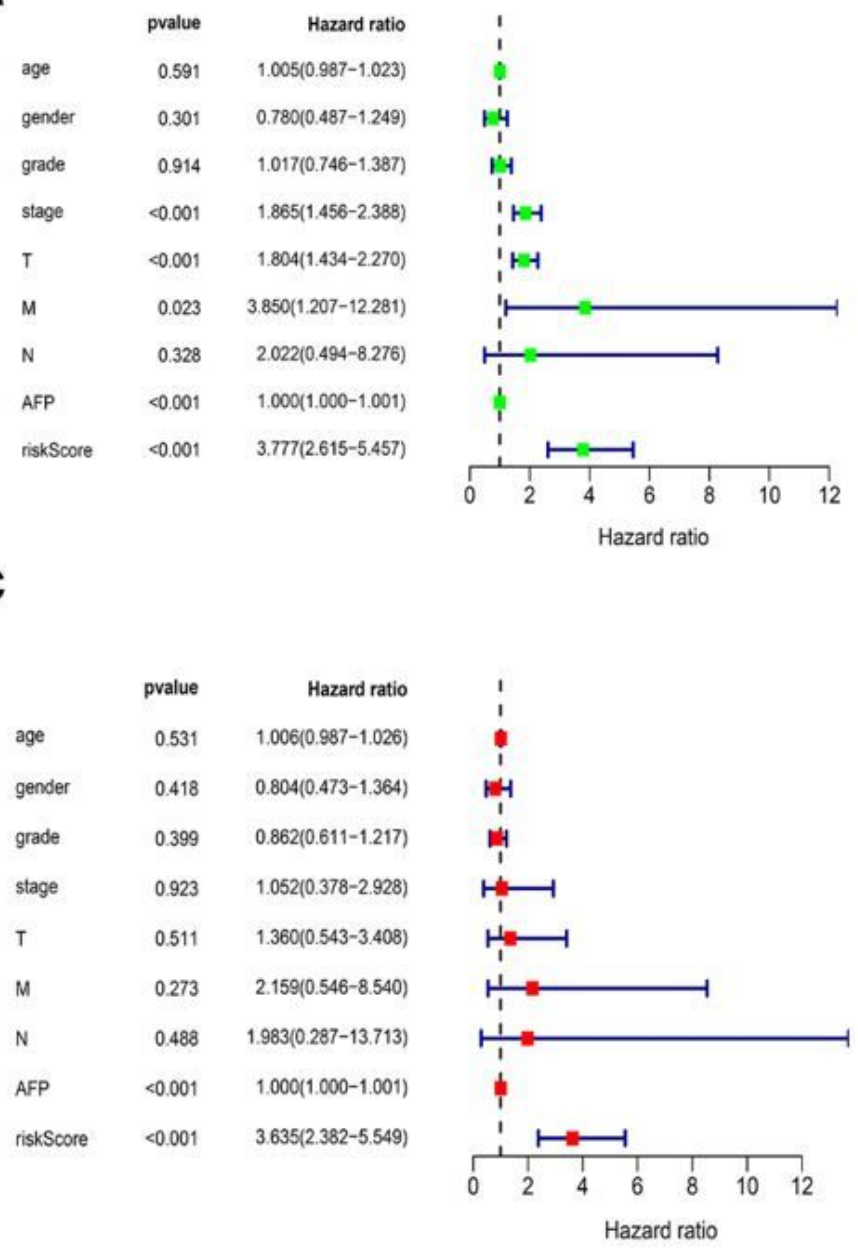

b

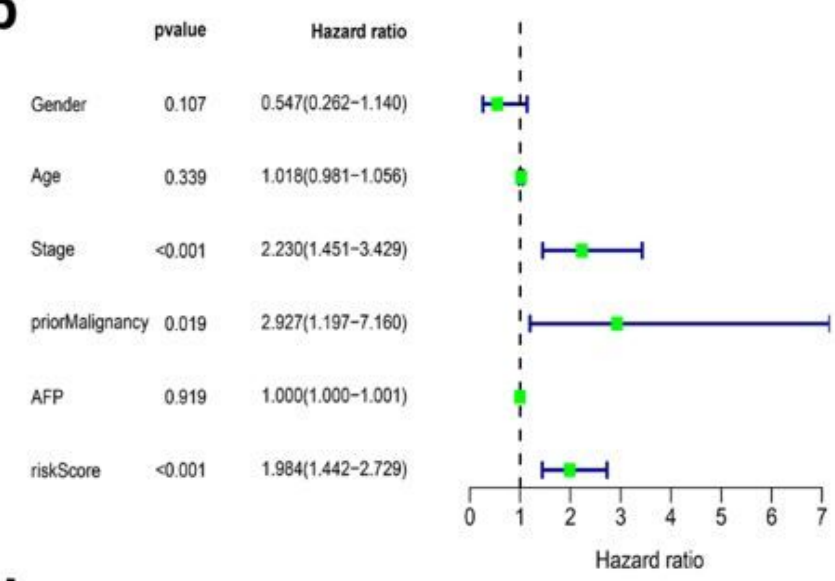

d
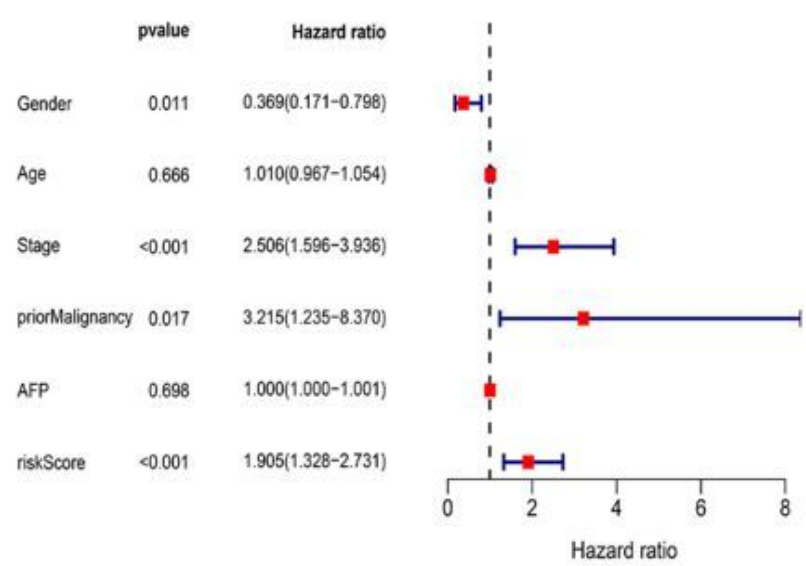

\section{Figure 3}

The metabolism related risk score is an independent prognostic factor for HCC. Univariate Cox regression analysis shows the prognostic value of risk scores and the other clinical variables in TCGA (a) and ICGC (c) cohorts. Multivariate Cox regression analysis of correlations between the risk score for OS and clinical variables. in TCGA (b) and ICGC (d) cohorts. Risk score shows significant prognostic value. 

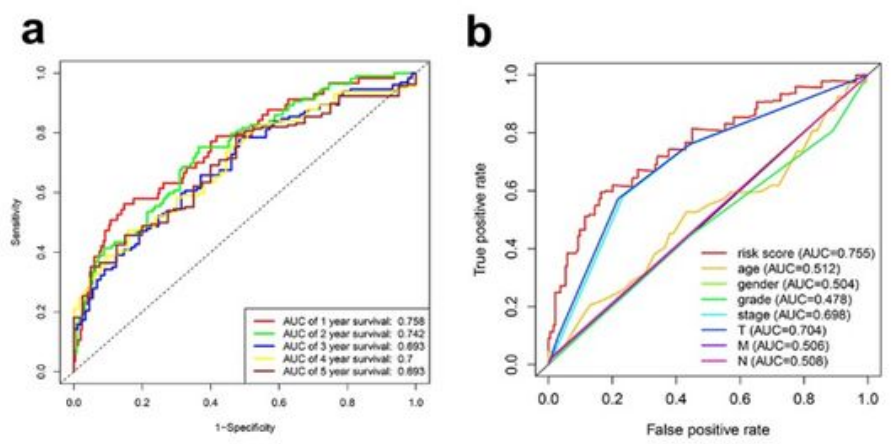

C Low risk - High risk
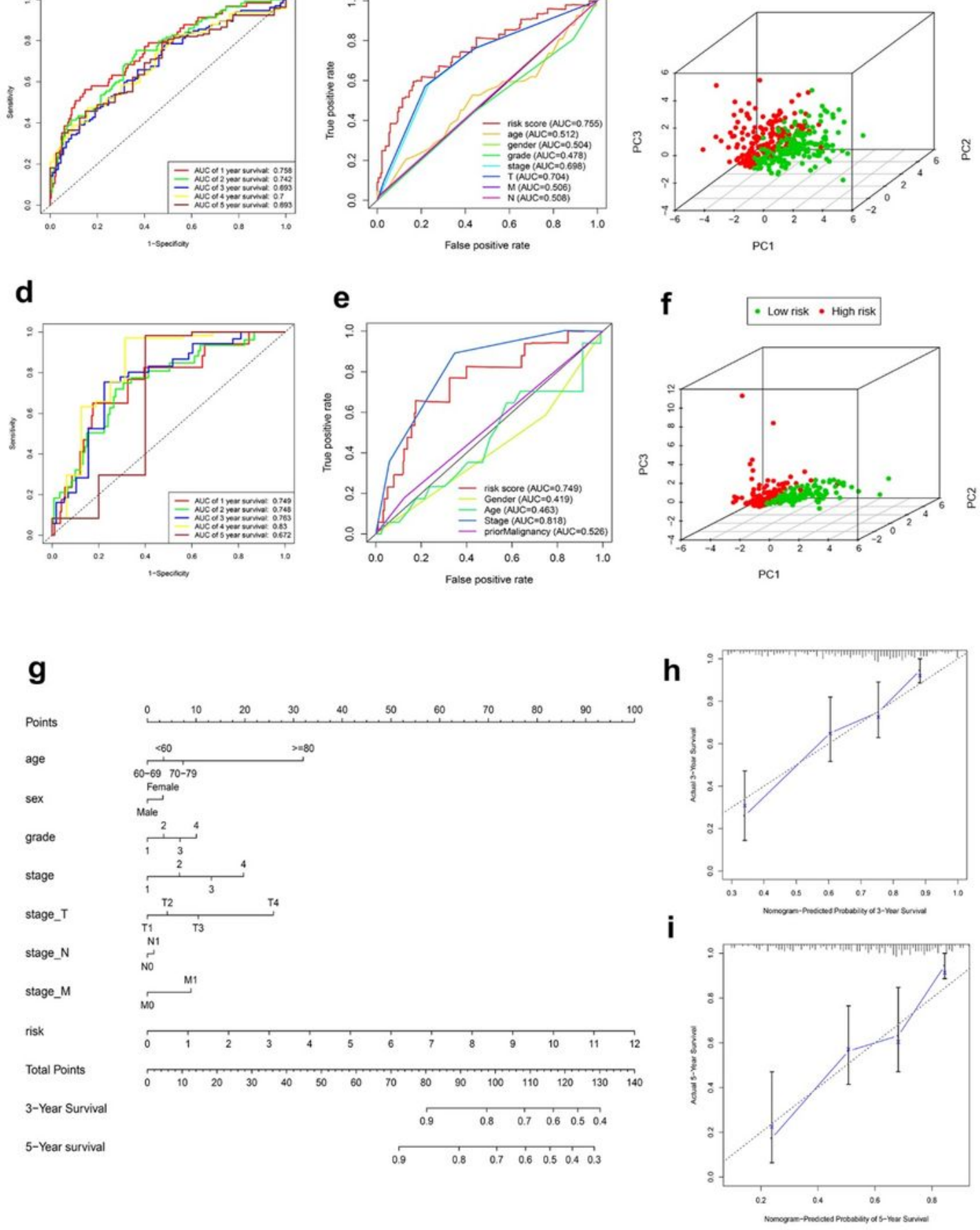

\section{Figure 4}

Evaluation of the metabolism-related prognostic model in TCGA and ICGC cohorts. (a, d) Time-dependent ROC curve analysis of the risk score at 1-, 2-, 3-,4- and 5-year for HCC. (b, e) ROC curve analysis of the risk score and other clinical variables including age, gender, grade, stage, T, M, N. (c, f) Principal Component Analysis (PCA) showed that risk score could distinguish HCC patients well. T, tumor size, M, metastasis, 
$\mathrm{N}$, regional lymph node. (g) Nomogram to predict the probability of OS for HCC in the TCGA cohort. (h,i) Calibration plot of the nomogram to predict the probability of OS at 3, 5 years in TCGA cohort.

a

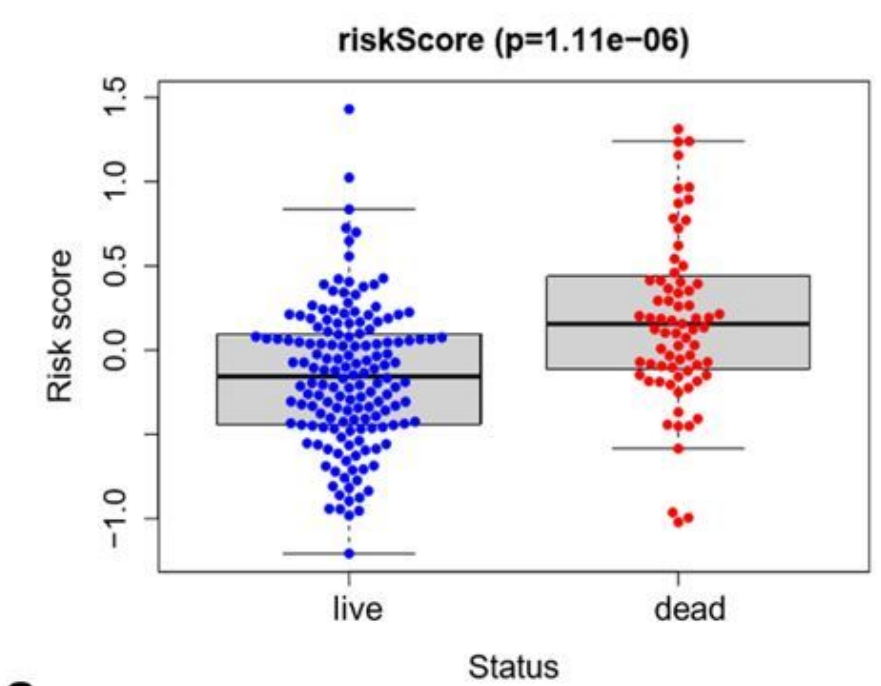

C

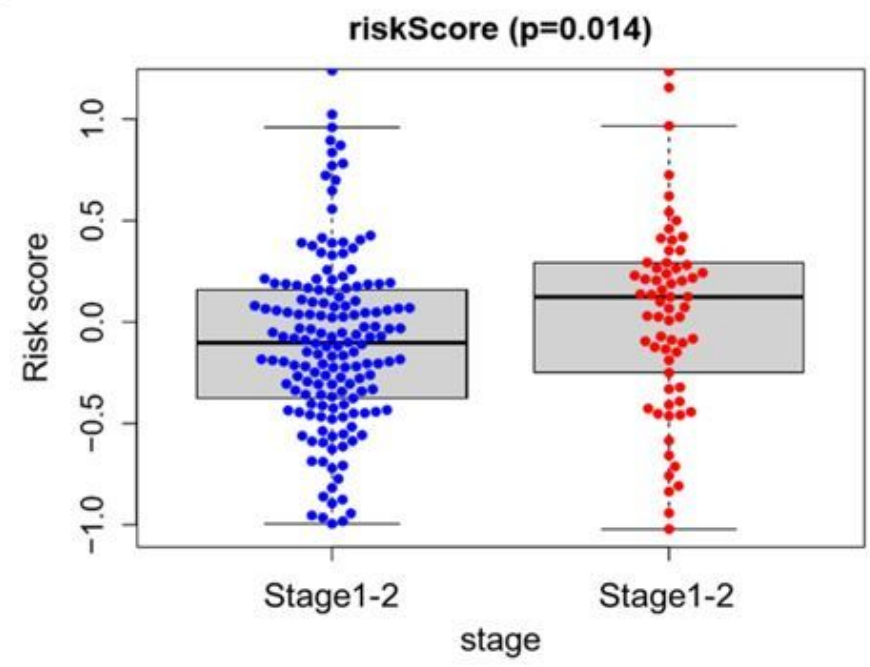

b

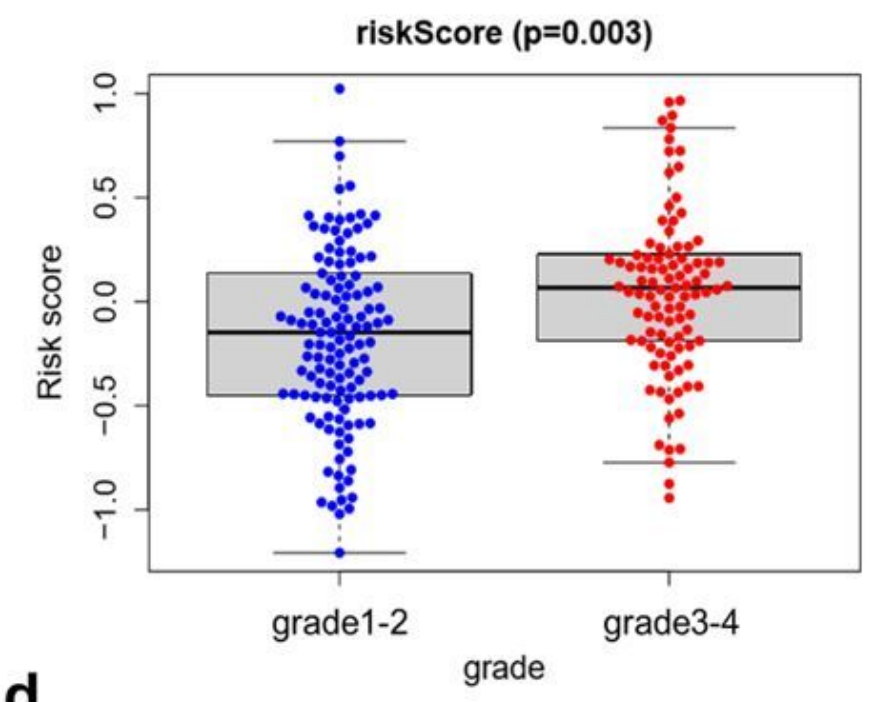

d

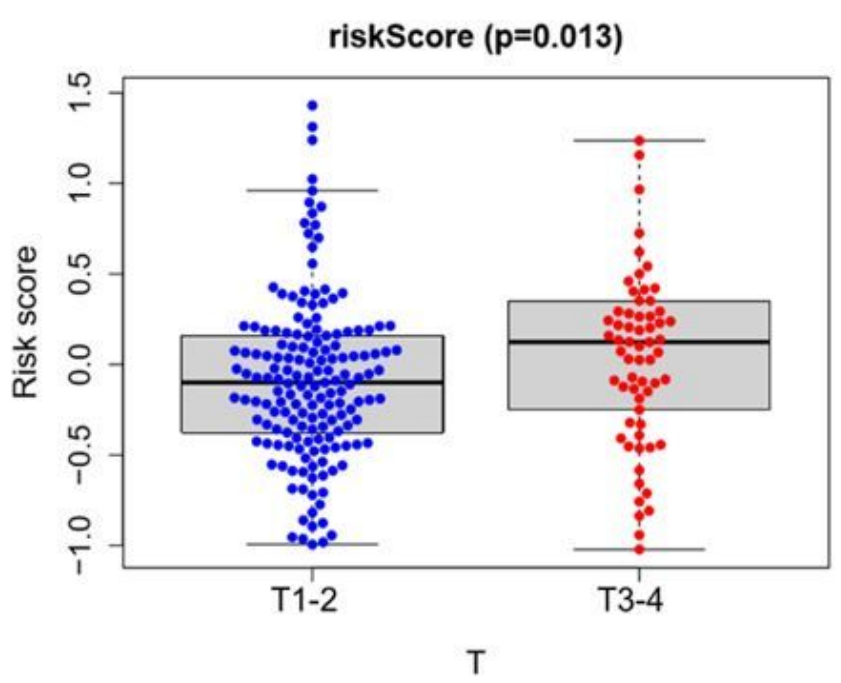

Figure 5

Clinical relevance of risk score in TCGA cohort. The risk score correlates with (a) status, (b) grade, (c) stage and (d) T stage in HCC. Risk score correlates with the progression of hepatocellular carcinoma and it is higher in patients with advanced hepatocellular carcinoma. T, tumor size. 

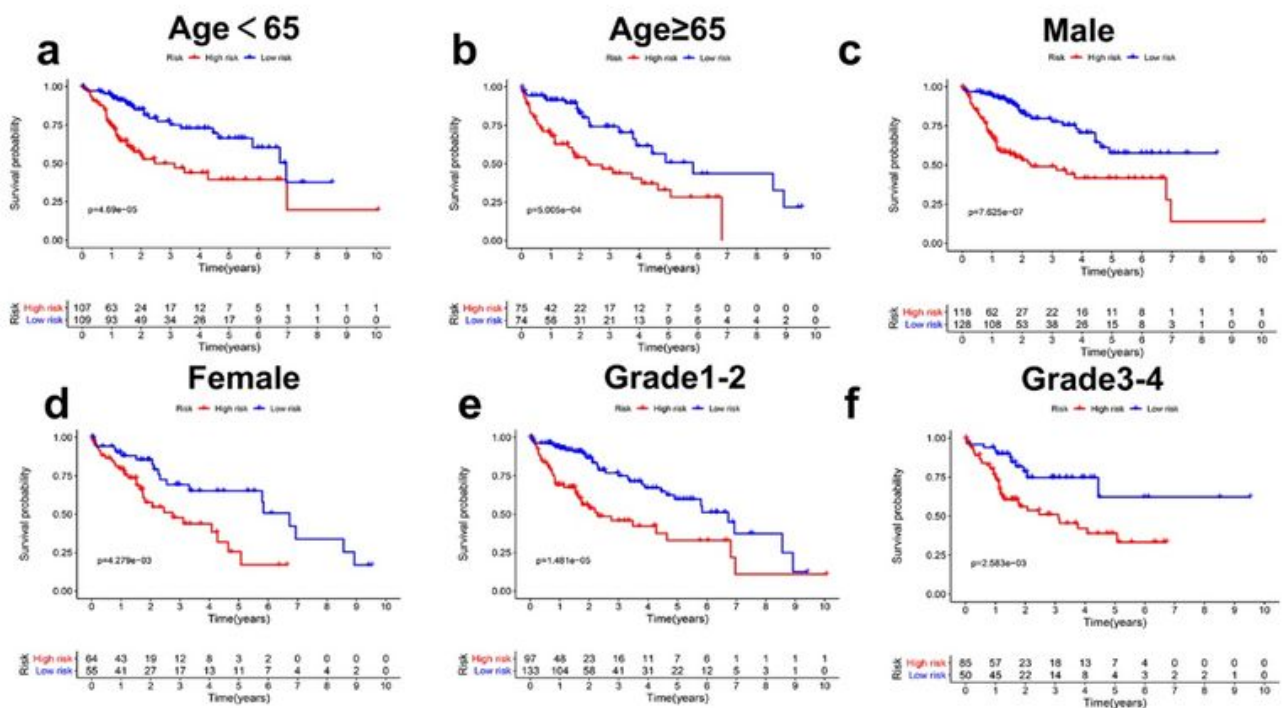

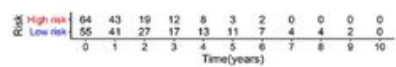
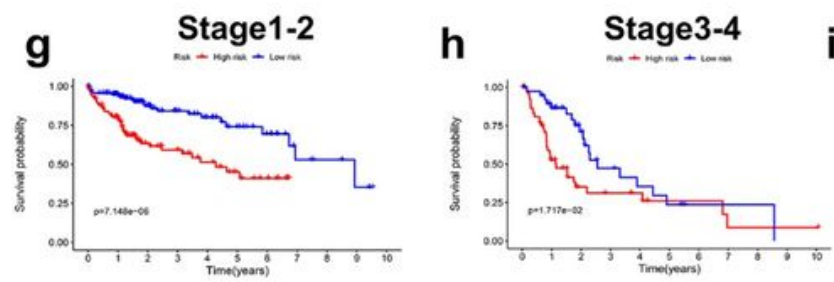

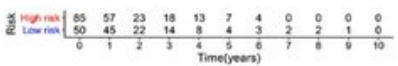
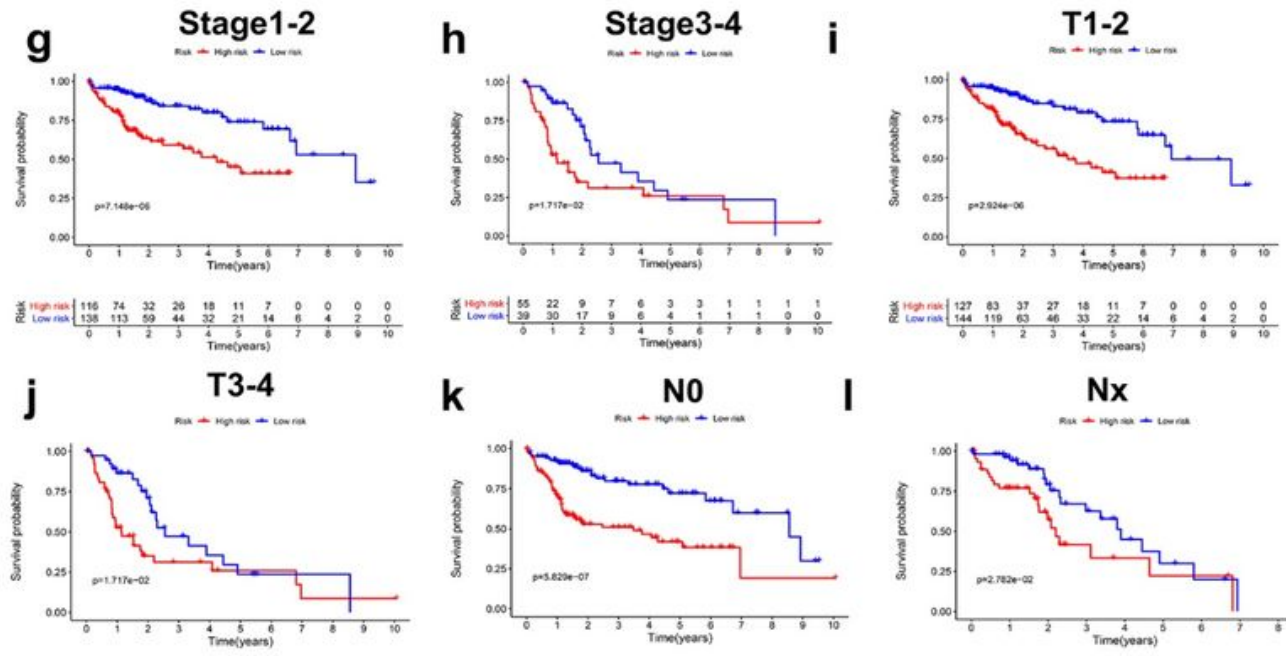

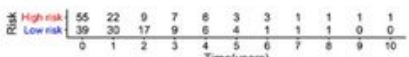
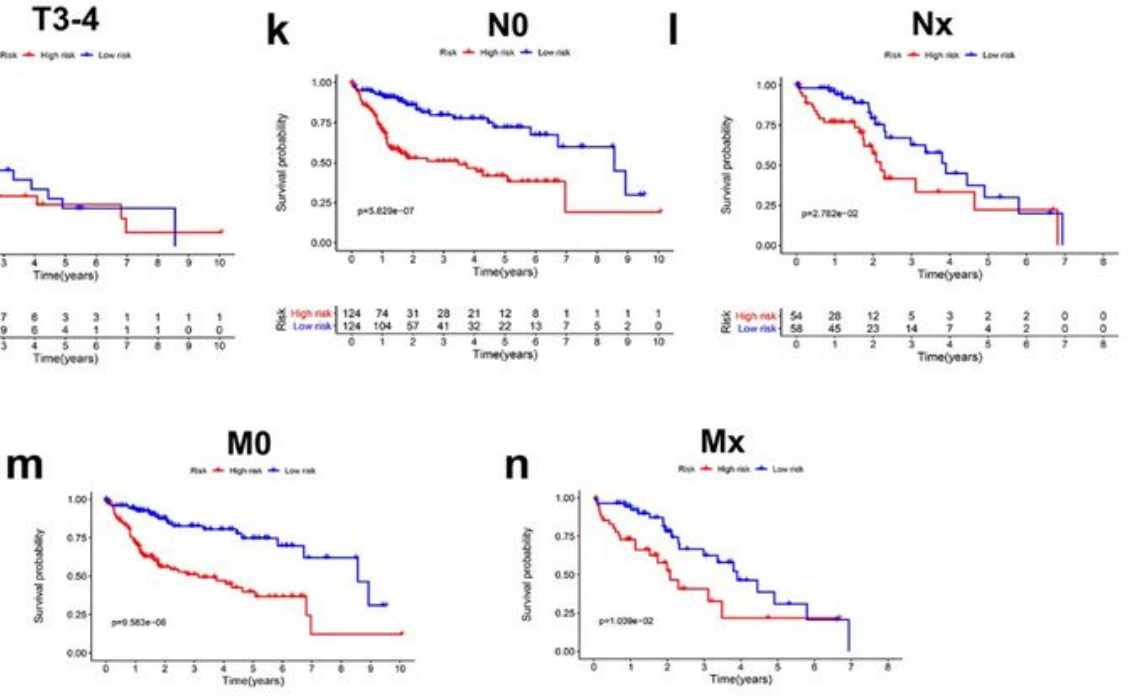

1)

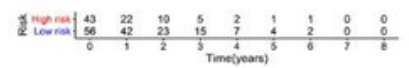

\section{Figure 6}

The correlation between risk score and other clinical features in the prognosis of HCC patients. $(a, b)$ Age. $(c, d)$ Gender. (e, f) Grade. $(g, h)$ stage. $(i, j)$ T stage. $(k, l)$ M stage. $(m, n) N$ stage. The OS time of the highrisk group is shorter than that of the low-risk group in HCC patients stratified by different clinical characteristics. $\mathrm{T}$, tumor size, $\mathrm{M}$, metastasis, $\mathrm{N}$, regional lymph node. 



Figure 7

Metabolism-related genes correlate with tumor immunity of HCC. $(a, b)$ Gene annotation enrichment analysis in high- and low- immune score groups show the potential association of metabolic reprogramming and tumor microenvironment.

\section{Supplementary Files}

This is a list of supplementary files associated with this preprint. Click to download.

- SupplementaryFigures.pptx

- SupplementaryTables.docx 